02

\title{
Резонансное возбуждение фотолюминесценции в кристаллогидрате уранилацетата
}

\author{
(C) В.С. Горелик ${ }^{1,2}$, А.А. Лобойко ${ }^{1,2}$, С.О. Нечипуренко ${ }^{1,3}$ \\ ${ }^{1}$ Физический институт им. П.Н. Лебедева РАН, \\ 119991 Москва, Россия \\ ${ }^{2}$ Московский государственный технический университет им. Н.Э. Баумана, \\ 105005 Москва, Россия \\ ${ }^{3}$ Московский физико-технический институт, \\ 141707 Долгопрудный, Россия \\ e-mail: gorelik@sci.lebedev.ru
}

Поступила в редакцию 03.10.2017 г.

\begin{abstract}
Предложен метод экспресс-анализа ураниловых соединений на основе резонансного волоконнооптического возбуждения фотолюминесценции (ФЛ) ультрафиолетовым излучением лазеров или полупроводниковых светодиодов. С использованием разработанной методики получены спектры ФЛ кристаллогидрата уранилацетата $\mathrm{UO}_{2}\left(\mathrm{CH}_{3} \mathrm{COO}\right)_{2} \cdot 2 \mathrm{H}_{2} \mathrm{O}$ при экспозициях $10^{-3} \mathrm{~s}$ предельно малого объема вещества $\left(10^{-9} \mathrm{~cm}^{3}\right)$. В качестве источников возбуждающего излучения использованы полупроводниковые светодиоды с длинами волн 369, 385, 410 и $466 \mathrm{~nm}$, а также импульсно-периодический азотный лазер с длиной волны генерации $337 \mathrm{~nm}$. Регистрация спектров ФЛ осуществлялась малогабаритным миниспектрометром, обеспечивающим возможность анализа спектров в диапазоне $200-1000 \mathrm{~nm}$.
\end{abstract}

DOI: $10.21883 /$ OS.2018.02.45528.218-17

\section{Введение}

Интерес к соединениям урана [1] в настоящее время обусловлен их применениями в атомной энергетике и возникающими вследствие этого проблемами переработки и утилизации. Интенсивные исследования спектров этих соединений ведутся $[2,3]$ с конца двадцатого столетия после начала прикладных работ в этой области [4]. Соединения урана представляют интерес также в связи с их особыми кристаллохимическими и физикохимическими свойствами $[5,6]$. Одним из таких свойств является наличие интенсивного люминесцентного свечения [7] в видимой области спектра, обусловленного электронным строением иона уранила $\left(\mathrm{UO}_{2}\right)^{+}[8]$.

$\mathrm{B}$ спектре электронных состояний этого иона имеется большое число энергетических переходов. Переходы находятся в ультрафиолетовой, инфракрасной и видимой областях. В монографии [9] приводятся общие сведения и анализ соединений ураниловой группы. Ранее в работах [10-17] были исследованы спектры фотолюминесценции (ФЛ) и поглощения органических и неорганических комплексов на основе уранила: силиката, перхлората, нитрата, сульфата, фторида и оксалата уранила при разных температурах. В этих работах было установлено, что спектры ФЛ ураниловых соединений при комнатной температуре обычно состоят из системы эквидистантно расположенных диффузных полос, занимающих область спектра около $450 \mathrm{~nm}$. В зависимости от условий регистрации обнаруживается 4-8 полос ФЛ с расстоянием между максимумами (в шкале частот) около $860 \mathrm{~cm}^{-1}$. Спектральные положения максимумов этих полос зависят от химического состава соответ- ствующего соединения. Охлаждение кристаллических образцов до температуры кипения жидкого кислорода или азота сопровождается расщеплением спектров ФЛ большинства ураниловых соединений в полосе ФЛ с полушириной $20-40 \mathrm{~cm}^{-1}$. В результате этого расщепления формируется структура спектров ФЛ при возбуждении различными длинами волн видимого и ультрафиолетового диапазонов $[10,18]$. Образовавшаяся структура индивидуальна для каждого соединения и очень чувствительна к наличию примесей.

В работах [19-23] приведен подробный анализ электронных конфигураций некоторых ураниловых соединений, а также детально описаны механизмы распределения энергии между собственными колебаниями кристалла. Экспериментальные результаты хорошо согласуются с результатами теоретико-группового анализа. Все ФЛ-исследования в этих работах, как правило, проводились при значительном охлаждении ураниловых комплексов.

В работе [24] на примере хлорида уранила было установлено, что областям сильного поглощения иона уранила соответствует возбуждающее излучение с частотами 20400 (490), 21000 (476), 21500 (465), 22500 (445), 23000 (434), 23900 (418), 24400 (410), $26000 \mathrm{~cm}^{-1}(384 \mathrm{~nm})$, а также с частотой, превышающей $27000 \mathrm{~cm}^{-1}(370 \mathrm{~nm})$.

Уранилацетат (наряду с уранилнитратом) является одним из основных урансодержащих соединений, встречаемых в природе [25]. В работе [26] проводились исследования оптических свойств, связанных со спектрами поглощения и ФЛ уранилацетата и уранилнитрата. В этой работе удалось зарегистрировать надежные спектры ФЛ 

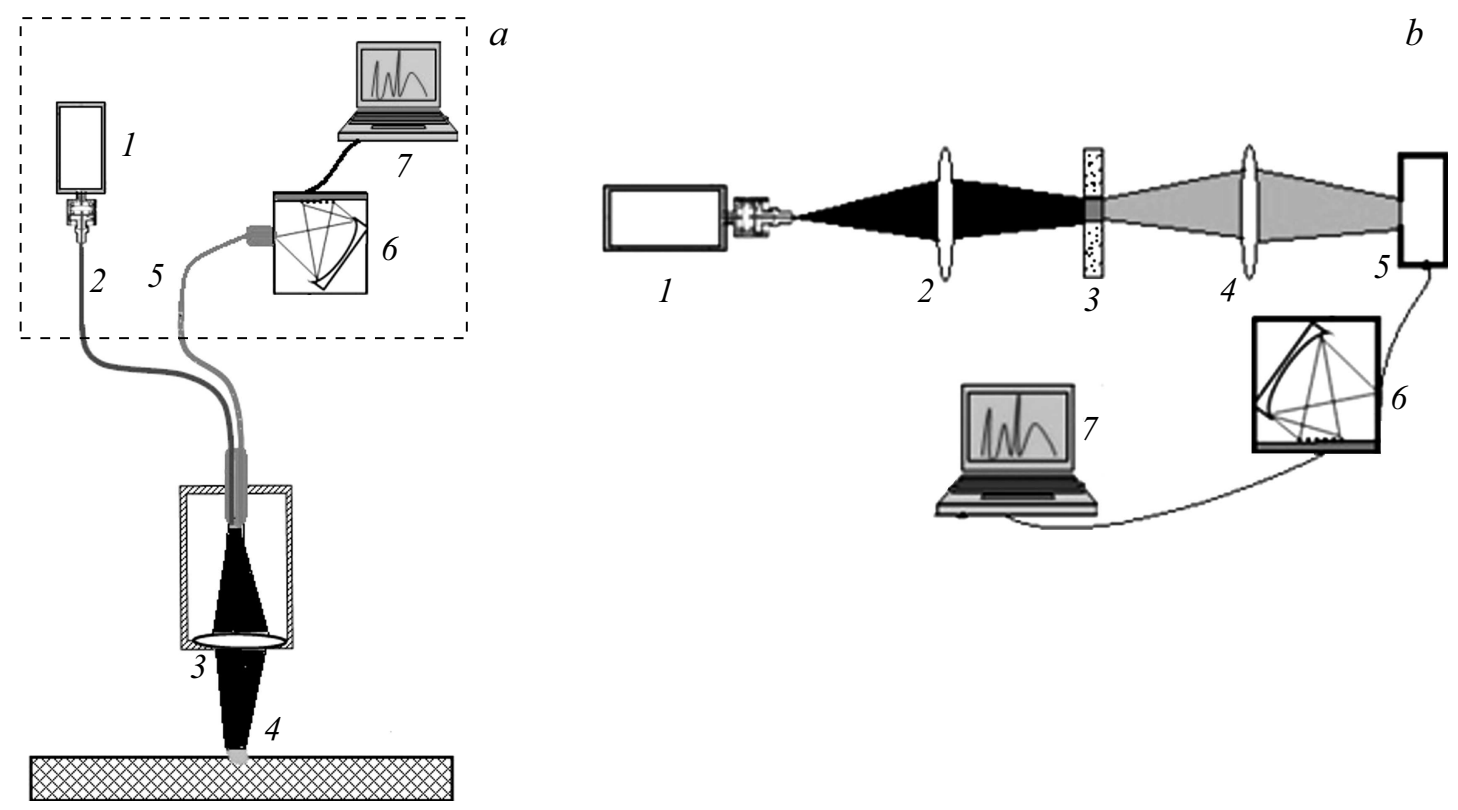

Рис. 1. Экспериментальная установка для возбуждения ФЛ в кристаллогидрате уранилацетата; $a$ : схема „на отражение“, 1 -источник оптического возбуждения, 2,5 - кварцевые волноводы, 3 - собирающая линза, 4 - кювета с исследуемым образцом, 6 - спектрометр, 7 - компьютер; $b$ : схема „на пропускание“, 1 - лазер, 2, 4 - собирающие линзы, 3 - образец, 5 фиксатор, 6 - спектрометр, 7 - компьютер.

только при низких температурах $(4-78 \mathrm{~K})$. Возбуждение ФЛ производилось при помощи ртутной лампы, излучающей на длине волны $365 \mathrm{~nm}$. У исследуемых образцов спектр ФЛ представлял собой четыре полосы с максимумами интенсивности на длинах волн 489, 507, 534 и $559 \mathrm{~nm}$, соответствующих переходам с первого возбужденного электронного уровня на колебательные подуровни ураниловой группы основного электронного состояния [27].

В работе [28] был зарегистрирован спектр ФЛ в кристаллах натрий-уранилацетата $\mathrm{NaUO}_{2}\left(\mathrm{CH}_{3} \mathrm{COO}\right)_{3}$ при комнатной температуре. При этом в качестве источников возбуждающего излучения применялись полупроводниковые светодиоды и лазер с длиной волны генерации $266 \mathrm{~nm}$. В этой работе было зарегистрировано излучение ФЛ с длинами волн 479, 498, 521 и $546 \mathrm{~nm}$. Также было обнаружено, что при импульсно-периодическом лазерном возбуждении твердотельным лазером с длиной волны $266 \mathrm{~nm}$ наблюдалось перераспределение интенсивности в спектре ФЛ на полосе, соответствующей $498 \mathrm{~nm}\left(21000 \mathrm{~cm}^{-1}\right)$. Авторами этой работы высказано предположение о возможности лазерной генерации в ионах уранила, аналогично генерации излучения на красителях [29].

Целью данной работы являлась разработка экспрессметода для выявления ураниловых компонент при комнатной температуре. На основе предложенной методики ставилась задача регистрации спектров ФЛ от поликристаллов кристаллогидрата уранилацетата $\mathrm{UO}_{2}\left(\mathrm{CH}_{3} \mathrm{COO}\right)_{2} \cdot 2 \mathrm{H}_{2} \mathrm{O}$ и его сопоставления с извест- ными спектрами ФЛ натрий-уранилацетата и некоторых других соединений.

\section{Методика эксперимента}

Уранилацетат существует в виде кристаллогидрата $\mathrm{UO}_{2}\left(\mathrm{CH}_{3} \mathrm{COO}\right)_{2} \cdot 2 \mathrm{H}_{2} \mathrm{O}$ [30]. Поликристалл относится к орторомбической сингонии и характеризуется пространственной группой симметрии $\operatorname{Pnam}\left(D_{2 h}^{16}\right)$ с параметрами решетки $Z=4, a=9.622, b=14.833$ и $c=6.808 \AA$. Линейные группы $\mathrm{UO}_{2}$ расположены вдоль оси $D_{2 h}$ точечной группы симметрии кристалла. Исследуемый образец представлял собой желтый порошок, помещенный в кварцевую кювету. Размер микрочастиц поликристаллов уранилацетата составлял около $100 \mu \mathrm{m}$. Исследования спектров ФЛ проводились при комнатной температуре.

В работе использовались две экспериментальные установки, представленные на рис. 1. В схеме „на отражение“ (рис. 1, $a$ ) излучение по кварцевому световоду диаметром $100 \mu \mathrm{m}$ направлялось на поверхность кристалла в направлении, близком к нормальному. ФЛ, возникающая в кристалле, попадала в другой световод [31].

Спектры ФЛ кристаллогидрата уранилацетата регистрировались при возбуждении квазимонохроматическими полупроводниковыми светодиодами с длинами волн

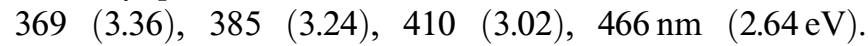
Спектр источников оптического возбуждения попадал в полосы сильного поглощения иона уранила [24,32]: 27000 (370), 26000 (384), 24400 (410) и $21500 \mathrm{~cm}^{-1}$ $(465 \mathrm{~nm})$. Возбуждающее излучение вследствие сильно- 

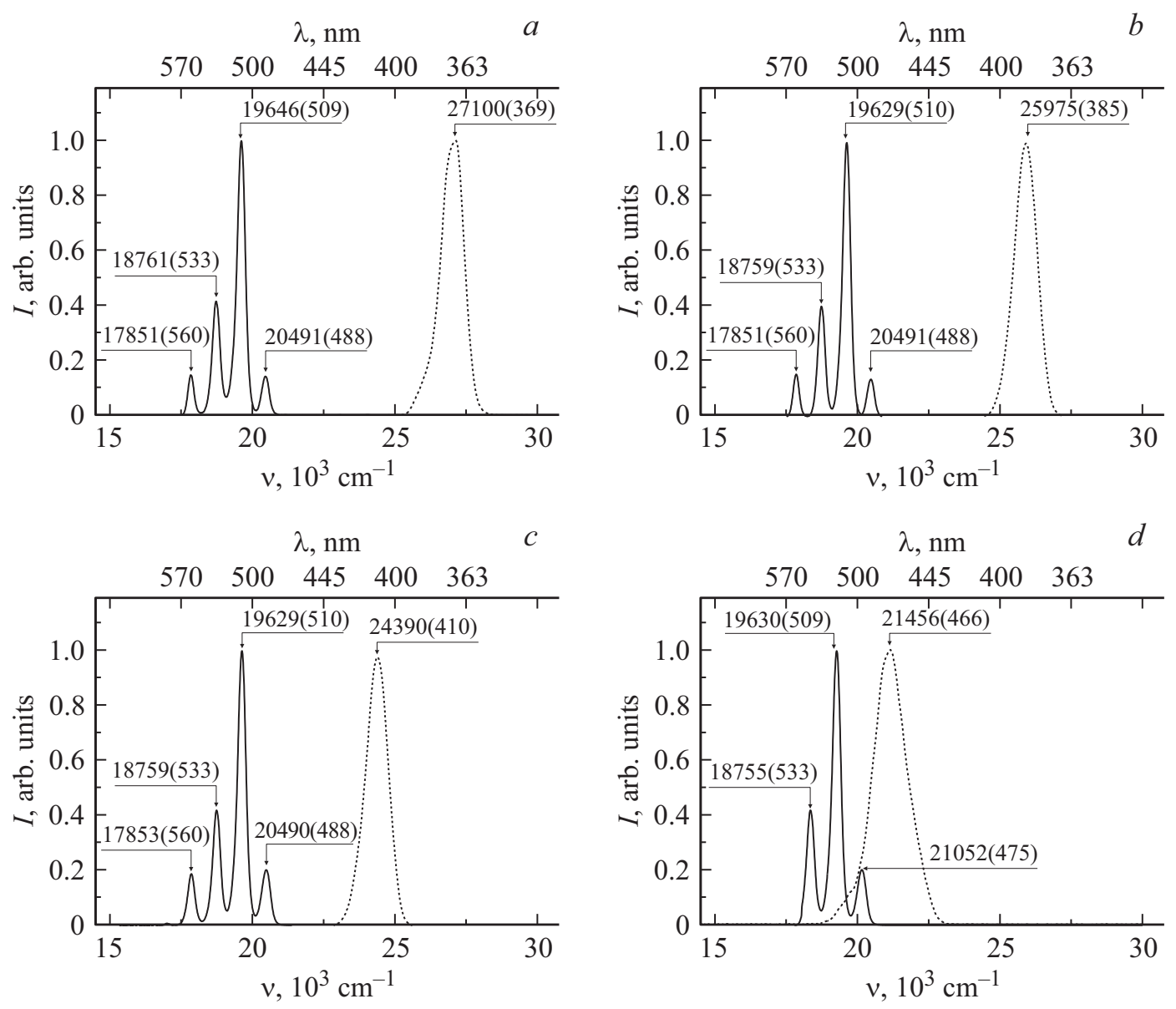

$\lambda, \mathrm{nm}$

e

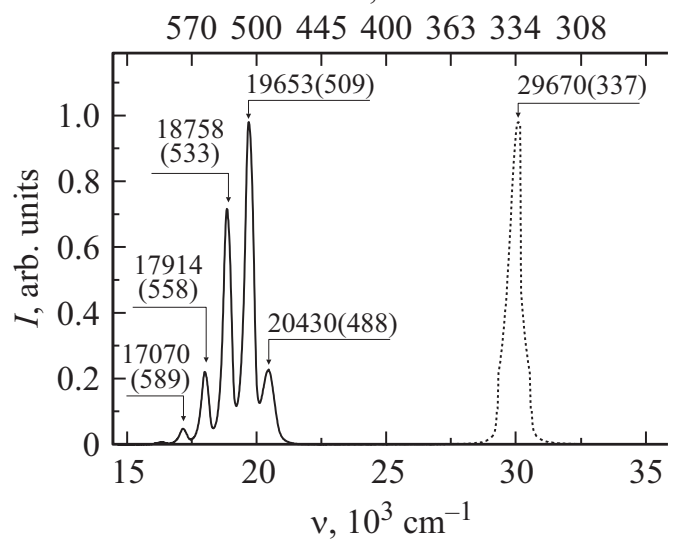

Рис. 2. Спектры ФЛ при возбуждении диодами с различными длинами волн: $a-\lambda=369 \mathrm{~nm}, b-385 \mathrm{~nm}, c-410 \mathrm{~nm}, d-466 \mathrm{~nm}$ и лазером: $e-\lambda=337 \mathrm{~nm}$.

го поглощения поликристаллов кристаллогидрата уранилацетата проникало на небольшую глубину образца порядка длины волны $\sim 10^{-5} \mathrm{~cm}$; при этом площадь освещаемой возбуждающим излучением поверхности образца составляла $\sim 10^{-4} \mathrm{~cm}^{2}$. Таким образом, объем люминесцирующего вещества составлял $\sim 10^{-9} \mathrm{~cm}^{3}$.

В схеме установки „на пропускание“ (рис $1, b)$ в качестве источника оптического возбуждения использовался азотный лазер на длине волны $337 \mathrm{~nm}$, работающий в импульсно-периодическом режиме. Спектр этого источника возбуждения также попадал в область сильного поглощения уранила $\left(>27000 \mathrm{~cm}^{-1}\right)$.

Полученные спектры ФЛ регистрировались цифровым спектрометром FSD-8, подключенным к компьютеру. Рабочий спектральный диапазон используемого спектрометра составлял 200-1000 nm при разрешении около $1 \mathrm{~nm}$. Экспозиции при регистрации спектров варьировались в диапазоне $0.1-128 \mathrm{~ms}$. 
Таблица 1. Спектральные положения максимумов интенсивности ФЛ кристаллогидрата $\mathrm{UO}_{2}\left(\mathrm{CH}_{3} \mathrm{COO}\right)_{2} \cdot 2 \mathrm{H}_{2} \mathrm{O}$ и их сопоставление с данными для $\mathrm{NaUO}_{2}\left(\mathrm{CH}_{3} \mathrm{COO}\right)_{3}$

\begin{tabular}{|c|c|c|c|c|c|c|c|c|c|c|}
\hline \multirow{2}{*}{$\begin{array}{c}\begin{array}{c}\text { Длина } \\
\text { волны, nm }\end{array} \\
337\end{array}$} & \multicolumn{5}{|c|}{$\begin{array}{c}\text { Частоты спектральных положений максимумов } \\
\text { интенсивности полос для } \mathrm{UO}_{2}\left(\mathrm{CH}_{3} \mathrm{COO}\right)_{2} \cdot 2 \mathrm{H}_{2} \mathrm{O}, \mathrm{cm}^{-1}\end{array}$} & \multicolumn{5}{|c|}{$\begin{array}{c}\text { Частоты спектральных положений максимумов } \\
\text { интенсивности полос для } \mathrm{NaUO}_{2}\left(\mathrm{CH}_{3} \mathrm{COO}\right)_{3}, \mathrm{~cm}^{-1}\end{array}$} \\
\hline & 17070 & 17914 & 18758 & 19653 & 20430 & - & - & - & - & - \\
\hline 369 & - & 17850 & 18761 & 19646 & 20491 & 17490 & 18301 & 19192 & 20099 & 20875 \\
\hline 385 & - & 17853 & 18759 & 19629 & 20491 & 17394 & 18315 & 19192 & 20035 & 20898 \\
\hline 410 & - & 17851 & 18759 & 19629 & 20490 & - & 18281 & 19192 & 20099 & 20875 \\
\hline 466 & - & - & 18755 & 19630 & 21052 & - & 18301 & 19192 & 20099 & 20875 \\
\hline
\end{tabular}

\section{Результаты экспериментальных исследований}

Полученные спектры ФЛ поликристаллов кристаллогидрата уранилацетата при возбуждении различными источниками оптического возбуждения приведены на рис. 2. На рис. $2, a-d$ возбуждение ФЛ осуществлялось полупроводниковыми светодиодами с длинами волн: $a-369 \mathrm{~nm}, b-385 \mathrm{~nm}, c-410 \mathrm{~nm}, d-466 \mathrm{~nm}$, на рис. 2,e - азотным лазером на длине волны $337 \mathrm{~nm}$. Из представленного рисунка видно, что наблюдаемые спектры ФЛ состоят из хорошо разрешенных четырех полос (рис. $2, a-c$ ) при возбуждении светодиодами и пяти полос при лазерном возбуждении (рис. 2,e). С уменьшением частоты возбуждающего излучения происходит изменение формы спектра ФЛ.

В табл. 1 представлены значения частот максимумов интенсивности $\left(\mathrm{B} \mathrm{cm}^{-1}\right)$ полос ФЛ для кристаллогидрата уранилацетата, полученными в данной работе. В этой же таблице для сравнения приводятся данные аналогичных измерений для натрий-уранилацетата [28].

\section{Обсуждение результатов эксперимента}

Таким образом, в данной работе предложен метод резонансного экспресс-анализа для обнаружения ураниловых комплексов. В основе метода лежит резонансное возбуждение иона уранила при попадании частоты возбуждающего излучения в область сильного поглощения образца. Такое возбуждение осуществимо с использованием ультрафиолетовых светодиодов, обеспечивающих излучение в узком спектральном диапазоне. Регистрация спектров ФЛ проводилась на основе оптоволоконных методов миниспектрометром типа FSD-8 от предельно малого объема образца $\left(10^{-9} \mathrm{~cm}^{3}\right)$ и при малых экспозициях $\sim 10^{-3} \mathrm{~s}$.

Полученные спектры ФЛ кристаллогидрата уранилацетата состояли из четырех хорошо разрешенных полос с максимумами интенсивности на частотах 17852, 18762 , 19630 и $20490 \mathrm{~cm}^{-1}$. Полученные спектры согласуются с результатами, полученными ранее [26] с использованием крупногабаритной установки и ртутной лампы в качестве источника возбуждающего излучения. Поглощение
Таблица 2. Номера и разности частот максимумов интенсивности ФЛ кристаллов $\mathrm{UO}_{2}\left(\mathrm{CH}_{3} \mathrm{COO}\right)_{2} \cdot 2 \mathrm{H}_{2} \mathrm{O}$ и $\mathrm{NaUO}_{2}\left(\mathrm{CH}_{3} \mathrm{COO}\right)_{3}$

\begin{tabular}{c|c|c|c}
\hline \multicolumn{2}{c|}{$\begin{array}{c}\text { Кристаллогидрат } \\
\text { уранилацетата } \\
\mathrm{UO}_{2}\left(\mathrm{CH}_{3} \mathrm{COO}\right)_{2} \cdot 2 \mathrm{H}_{2} \mathrm{O}\end{array}$} & \multicolumn{2}{|c}{$\begin{array}{c}\text { Натрий-уранилацетат } \\
\mathrm{NaUO}_{2}\left(\mathrm{CH}_{3} \mathrm{COO}\right)_{3}\end{array}$} \\
\hline $\begin{array}{c}v \pm 50, \mathrm{~cm}^{-1} \\
\text { (положение } \\
\text { максимумов } \\
\text { интенсивности ФЛ) }\end{array}$ & $\begin{array}{c}\Delta v \pm 50, \\
\mathrm{~cm}^{-1}\end{array}$ & $\begin{array}{c}v \pm 50 \mathrm{~cm}^{-1} \\
\text { (положение } \\
\text { максимумов } \\
\text { интенсивности ФЛ })\end{array}$ & $\begin{array}{c}\Delta v \pm 50, \\
\mathrm{~cm}^{-1}\end{array}$ \\
\hline 17851 & 906 & 18300 & 892 \\
18757 & 873 & 19192 & 907 \\
19630 & 861 & 20099 & 776 \\
\hline
\end{tabular}

и испускание света в ионах уранила иллюстрирует диаграмма энергетических уровней [33] кристаллогидрата уранилацетата, приведенная на рис. 3. На этой диаграмме основное и возбужденное электронные состояния обозначены $S_{0}$ и $S_{1}$. Каждый из этих энергетических уровней состоит из набора колебательновращательных подуровней, обозначенных 0,1,2 ит.д. Поглощение иона уранила $\left(\mathrm{UO}_{2}\right)^{+}$соответствует переходам с самого нижнего основного электронного состояния $S_{0}$ на возбужденное $S_{1}$, а также на его подуровни.

Наблюдаемые спектры ФЛ соответствуют [27] излучательным переходам с энергетического уровня $S_{1}$ иона уранила в основное энергетическое состояние $S_{0}$ или на его колебательные подуровни основного состояния. Таким образом, наибольшая частота излучательного перехода $v_{1}=20490 \mathrm{~cm}^{-1}$ соответствует резонансной частоте перехода электрона на основной электронный уровень. Переходы с частотами $v_{2}=19630, v_{3}=18762$, $v_{4}=17852 \mathrm{~cm}^{-1}$ соответствуют переходам с возбужденного электронного состояния в основное - на первый, второй и третий колебательные подуровни соответственно. В табл. 2 приведены частоты максимумов 
Таблица 3. Сопоставление частот максимумов интенсивности ФЛ комплексов на основе уранила в $\mathrm{cm}^{-1}$

\begin{tabular}{l|c|c|c|c|c|c|c}
\hline \multicolumn{1}{c|}{ Соединение } & $\begin{array}{c}v_{1}, \mathrm{~cm}^{-1} \\
(1 \text { пик })\end{array}$ & $\begin{array}{c}v_{2}, \mathrm{~cm}^{-1} \\
(2 \text { пик })\end{array}$ & $\begin{array}{c}v_{3}, \mathrm{~cm}^{-1} \\
(3 \text { пик })\end{array}$ & $\begin{array}{c}v_{4}, \mathrm{~cm}^{-1} \\
(4 \text { пик })\end{array}$ & $\begin{array}{c}v_{5}, \mathrm{~cm}^{-1} \\
(5 \text { пик })\end{array}$ & $\begin{array}{c}v_{6}, \mathrm{~cm}^{-1} \\
(6 \text { пик })\end{array}$ & $\begin{array}{c}v_{7}, \mathrm{~cm}^{-1} \\
(7 \text { пик })\end{array}$ \\
\hline Силикат уранила $\mathrm{K}\left(\mathrm{UO}_{2}\right) \cdot\left(\mathrm{SiO}_{3} \mathrm{OH}\right) \cdot 15 \mathrm{H}_{2} \mathrm{O}_{5}[17]$ & 19787 & 18685 & 17921 & 17259 & - & - & - \\
Фторид $\mathrm{UO}_{2} \mathrm{~F}_{2}[11]$ & 20234 & 19366 & 18500 & 17490 & 16640 & - & - \\
Чистый уранил $\mathrm{UO}_{2}[13]$ & 20338 & 19459 & 18574 & 17746 & 16895 & - & - \\
Перхлорат $\mathrm{UO}_{2}\left(\mathrm{ClO}_{4}\right) \cdot 6 \mathrm{H}_{2} \mathrm{O}[15]$ & 20408 & 19342 & 18691 & - & - & - \\
Кристаллогидрат уранилацетата $\mathrm{UO}_{2}\left(\mathrm{CH}_{3} \mathrm{COO}\right)_{2} \cdot 2 \mathrm{H}_{2} \mathrm{O}$ & 20491 & 19630 & 18757 & 17851 & - & - \\
{$[$ настоящая работа] } & & & & & - & - \\
Нитрат $\mathrm{UO}_{2}\left(\mathrm{NO}_{3}\right)_{2}[12]$ & 20703 & 19801 & 19011 & 18083 & 17152 & - & - \\
Натрий-уранилацетат $\mathrm{NaUO}_{2}\left(\mathrm{CH}_{3} \mathrm{COO}\right)_{3}[28]$ & 20875 & 20099 & 19192 & 18300 & - & - & - \\
Сульфат $\mathrm{K}_{2}\left(\mathrm{UO}_{2}\right) \cdot\left(\mathrm{SO}_{4}\right)_{2}[10]$ & 20986 & 20325 & 19493 & 18657 & 17838 & 17004 & 16155
\end{tabular}

интенсивности ФЛ для кристаллогидрата уранилацетата и натрий-уранилацетата. По данным этой таблицы можно сделать вывод, что наблюдаемые полосы ФЛ являются эквидистантными (с точностью до ошибки измерений). Видно, что эти максимумы на шкале частот (а следовательно, и колебательные уровни энергии) отстоят друг от друга примерно на $870 \pm 50 \mathrm{~cm}^{-1}$. Эта частота соответствует симметричным валентным колебаниям иона уранила [27]. Значения соответствующих частот положений максимумов интенсивности ФЛ для $\mathrm{UO}_{2}\left(\mathrm{CH}_{3} \mathrm{COO}\right)_{2} \cdot 2 \mathrm{H}_{2} \mathrm{O}$ и $\mathrm{NaUO}_{2}\left(\mathrm{CH}_{3} \mathrm{COO}\right)_{3}$ отличаются на величину $400 \mathrm{~cm}^{-1}$. Эти отличия связаны с положением первого возбужденного электронного уровня. Резонансная частота перехода для кристаллогидрата уранилацетата $\left(20491 \mathrm{~cm}^{-1}\right)$ на $384 \mathrm{~cm}^{-1}$ меньше чем у натрий-уранилацетата $\left(20875 \mathrm{~cm}^{-1}\right)$. Таким образом, положение первого электронного возбужденного уровня у натрий-уранилацетата находится выше, чем у кристаллогидрата уранилацетата на величину $384 \mathrm{~cm}^{-1}$. Эти уровни соответствуют возбуждению иона уранила в конденсированных средах, а также зависят от лигандов, входящих в его среду.

В табл. 3 приводятся значения частот $\left(\right.$ в $\left.\mathrm{cm}^{-1}\right)$ максимумов ФЛ для некоторых упомянутых во введении комплексов на основе уранила. Из этой таблицы видно, что можно однозначно детектировать кристаллогидрат уранилацетата по ФЛ-свойствам при комнатной температуре. С учетом малых габаритов созданной экспериментальной установки может быть изготовлен портативный экспресс-анализатор для исследования окружающей среды на наличие ураниловых компонент. Резонансное возбуждение иона $\left(\mathrm{UO}_{2}\right)^{+}$и возбуждение источниками спектральной области, соответствующей сильному электронному поглощению иона уранила, обеспечивают эффективную люминесценцию кристалла. При этом полоса ФЛ с максимумом на частоте $19630 \mathrm{~cm}^{-1}$ является самой интенсивной. Эта полоса представляет собой узкий пик. Ее ширина не превышает $20 \mathrm{~nm}$. Проявление узкого интенсивного пика свидетельствуют о высокой доле вероятности испускания фотона с частотой $19630 \mathrm{~cm}^{-1}$ при ФЛ.

Следует отметить, что в работе [28] при возбуждении ФЛ натрий-уранилацетата под действием импульсно-периодического лазерного возбуждения твердотельным лазером с длиной волны $266 \mathrm{~nm}$ при накачке по четырехуровневой схеме наблюдалось явление суперлюминесценции. Суперлюминесценция проявлялась в усилении интенсивности ФЛ в полосе, соответствующей $498 \mathrm{~nm}\left(20100 \mathrm{~cm}^{-1}\right)$.

Наблюдение суперлюминесценции в кристаллах натрий-уранилацетата свидетельствует о возможности лазерной генерации и в кристаллогидрате уранилацетата $\mathrm{UO}_{2}\left(\mathrm{CH}_{3} \mathrm{COO}\right)_{2} \cdot 2 \mathrm{H}_{2} \mathrm{O}$. В отличие от известных лазеров на красителях [34] источники, использующие эффект суперлюминесценции в ураниловых соединениях, характеризуются большим коэффициентом усиления. Усиление интенсивности в кристаллогидрате уранилацетата должно проявляться на частоте $19630 \mathrm{~cm}^{-1}$ (вследствие отличий положений первых возбужденных уровней на величину $385 \mathrm{~cm}^{-1}$ ). При этом в качестве источника накачки предлагается использовать твердотельный лазер с длиной волны $266 \mathrm{~nm}$.

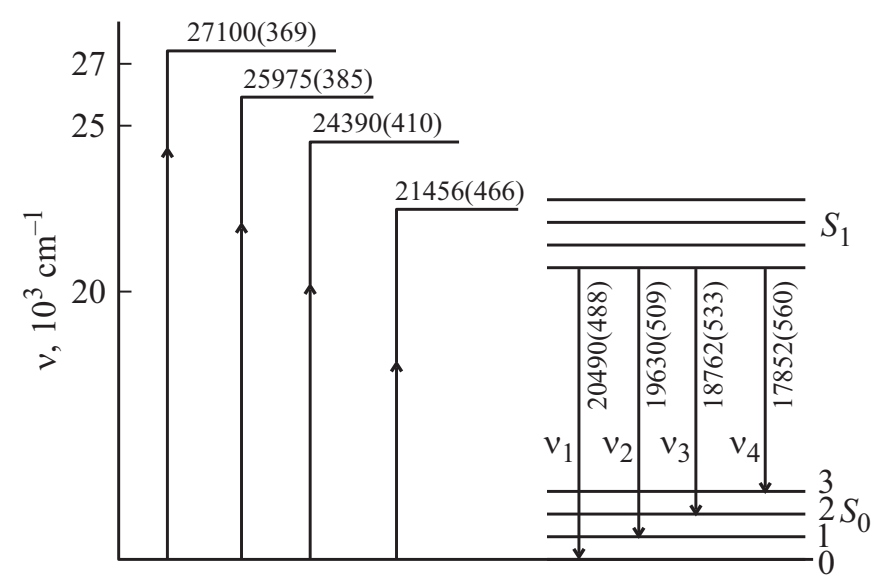

Рис. 3. Диаграмма энергетических уровней кристалла $\mathrm{UO}_{2}\left(\mathrm{CH}_{3} \mathrm{COO}\right)_{2} \cdot 2 \mathrm{H}_{2} \mathrm{O}$. 


\section{Заключение}

Полученные результаты свидетельствуют о возможности проведения экспресс-анализа небольших количеств кристаллогидрата уранилацетата в окружающей среде методом волоконно-оптического возбуждения спектров ФЛ ультрафиолетовыми и синими полупроводниковыми светодиодами. Установлено, что в спектре ФЛ присутствуют четыре полосы с положениями максимумов интенсивности на частотах 17851, 18757, 19630 и $20491 \mathrm{~cm}^{-1}$. Наблюдаемые спектры ФЛ, полученные по схеме „на отражение“, соответствуют излучательным переходам с возбужденных энергетических электронных уровней иона уранила в основное энергетическое состояние и на колебательные подуровни, расположенные эквидистантно $\left(860 \mathrm{~cm}^{-1}\right)$. Выявлена аналогичная спектральная картина в схеме „на пропускание“ при импульсно-периодическом лазерном возбуждении. Установлена близость спектров ФЛ для кристаллогидрата уранилацетата и натрий-уранилацетата. Вибронная структура в этих спектрах обусловлена проявлением симметричных колебаний иона уранила ( $A_{1}$-типа). Наблюдаемые различия связаны с различным положением первого электронного возбужденного уровня в обсуждаемых соединениях. В натрий-уранилацетате это положение находится выше, чем у кристаллогидрата уранилацетата, на $385 \mathrm{~cm}^{-1}$. Установлена возможность лазерной генерации в кристаллах $\mathrm{UO}_{2}\left(\mathrm{CH}_{3} \mathrm{COO}\right)_{2} \cdot 2 \mathrm{H}_{2} \mathrm{O}$, аналогичной генерации на красителях при накачке по четырехуровневой схеме.

Работа выполнена при поддержке РФФИ, грант № 1652-00026 (БРФФИ-РФФИ Ф16Р-063).

\section{Список литературы}

[1] Audi G., Bersillon O., Blachot J., Wapstra A. // Nucl. Phys. A. 2003. V. 729. P. 3.

[2] Bostick B.C., Fendorf S., Barnett M.O., Jardine P.M., Brooks S.C. // Soil Sci. Soc. Am. 2002. V. 66. P. 99.

[3] Barraclough C.G., Cockman R.W., O'Donnell T.A. // Inorg. Nucl. Chem. Lett. 1981. V. 17. P. 83.

[4] Komura K., Yamamoto M., Ueno K. // Nuclear Inst. and Methods in Physics Research A. 1990. V. 295. P. 461.

[5] Gaziev S.A., Gorshkov N.G., Mashirov L.G., Suglobov D.N. // Inorg. Chem. Acta. 1987. V. 139. P. 345.

[6] Denning R.G., Snellgrove T.P., Woodwark D.R. // Theor. Mol. Phys. 1979. V. 37. P. 1109.

[7] Meinrath G. // J. Radioanal. Nucl. Chem. 1997. V. 224. P. 119.

[8] Liu G. // J. Phys. Chem. A. 2011. V. 115. P. 12419.

[9] Rabinowitch E., Belford R.L. Spectroscopy and Photochemistry of Uranyl Compounds. N.Y.: The Macmillan Co. 1964.375 p.

[10] Nichols E.L., Howes H.L. Fluorescence of the Uranyl Salts. Carnegie Inst.: Wash. Pub., 1919. 298 p.

[11] Dieke G.H., Duncan A.B.F. Spectroscopic Properties of Uranium Compounds. N.Y.: McGraw-Hill Book Co., 1949. $290 \mathrm{p}$.
[12] Sevchenko A.N., Vdovenko V.M., Kovaleva T.V. // Zhur. Eksptl. Teoret. Fiz. 1951. V. 21. P. 204.

[13] Wang Z., Zachara J.M., Yantasee W., Gassman P.L., Liu Ch., Joly A. // Environ. Sci. Technol. 2004. V. 38. P. 5591.

[14] Flint C.D., Tanner P.A. // J. Chem. Soc. 1978. V. 74. P. 2210.

[15] Pant D.D., Khandelwal D.P. // Sei. Industr. Res. B. 1959. V. 18. P. 126.

[16] Mizuoka K., Tsushima S., Hasegawa M., Hoshi T., Ikeda Y. // Inor. Chem. 2005. V. 44. P. 6211.

[17] Wang Z., Zachara J.M., Gassman P.L., Liu Ch., Qafoku O., Yantasee W., Catalano J.G. // Geochimica et Cosmochimica Acta. 2005. V. 69. P. 1391.

[18] Leung A., Hayashibara L., Spadaro J. // J. Phys. Chem. Solids. 1999. V. 60. P. 299.

[19] Denning R.G. // J. Phys. Chem. A. 2007. V. 111. P. 4125.

[20] Denning R.G., Snellgrove T.R., Woodwark D.R. // Molec. Physics. 1975. V. 30. P. 1819.

[21] Flint C.D., Sharma P. // Molec. Chem. Phys. 1983. V. 79. P. 317.

[22] Flint C.D., Tanner P.A. // Molec. Chem. Phys. 1982. V. 78. P. 103.

[23] Denning R.G., Morrison I.D. // Chem. Phys. Lett. 1991. V. 180. P. 101.

[24] Christiane Görller-Walrand, Sandy De Houwer // Phys. Chem. 2004. V. 6. P. 3292.

[25] Lide D.R. Handbook of Chemistry and Physics. Boca Raton. FL: CRC Press, 1998. 566 p.

[26] Volod'ko L.V., Turetskaya E.A. // J. Appl. Spectr. 1965. V. 3. P. 180.

[27] Burrows H.D., Kemp T.J. // Chem. Soc. Rev. 1974. V. 3. P. 139.

[28] Горелик В.С., Коршунов В.М., Войнов Ю.П. // Опт. и спектр. 2016. Т. 121. № 6. С. 881.

[29] Бабенко В.А., Малышев В.И., Сычев А.А., Шибанов А.Н. // Квант. электрон. 1975. Т. 2. № 9. С. 1923.

[30] Howatson J., Grev D.M., Morosin B. // J. Inorg. Nucl. Chem. 1975. P. 1933.

[31] Горелик В.С., Литвинова А.О., Умаров М.Ф. // Кратк. сообщ. по физ. Физ. инст. им. П.Н. Лебедева РАН. 2014. № 11 . C. 3 .

[32] Xiaoping Sun, Kolling D.R.J., Hajer Mazagri // Inorg. Chim. Acta. 2015. V. 435. P. 117.

[33] Jablonski A. // Nature. 1933. V. 131. P. 839.

[34] Liu G.K., Zhuang H.Z., Beitz J.V. // Phys. Solid State. 2002. V. 44. P. 1433. 